\title{
Effect of Site Specific Nutrient Management for Targeted Yields on Population Dynamics of Sucking Pests in Bt-Cotton (Gossypium hirsutum L.)
}

\author{
Vinayak Hosamani $^{*}$, B.M. Chittapur ${ }^{2}$, Mallikarjun ${ }^{3}$, A.S. Halepyati ${ }^{4}$, \\ Satyanarayana Rao ${ }^{5}$, M.B. Patil ${ }^{6}$, N.L. Rajesh $^{7}$ and Venkatesh Hosamani ${ }^{8}$ \\ ${ }^{1}$ P2 BSF, Nagenahally, Kunigal, Central Silk Board, Bangalore/ UAS, Raichur, \\ Karnataka, India \\ ${ }^{2}$ Directorate of Extension, ${ }^{3} e-S A P$, Project, University of Agricultural Sciences, \\ Raichur, Karnataka, India \\ ${ }^{4}$ University of Agricultural Sciences, Raichur, Karnataka, India \\ ${ }^{5}$ Research Institute on Organic Farming, MARS. UAS, Raichur, Karnataka, India \\ ${ }^{6}$ AEEC, Koppal, UAS, Raichur, Karnataka, India \\ ${ }^{7}$ (SS\&AC), COA, UAS, Raichur, Karnataka, India \\ ${ }^{8}$ Entomology, COH, Munirabad-Koppal, Karnataka, India \\ *Corresponding author
}

\section{A B S T R A C T}

\begin{tabular}{|l|}
\hline K e y w o r d s \\
$\begin{array}{l}\text { Bt cotton, SSNM and } \\
\text { RDF, Cotton, Leaf } \\
\text { hopper, Thrips, Aphids, } \\
\text { Population dyanamics } \\
\text { and Sucking pest }\end{array}$ \\
\hline Article Info \\
\hline $\begin{array}{l}\text { Accepted: } \\
\text { 22 July } 2018 \\
\text { Available Online: } \\
\text { 10 August } 2018\end{array}$ \\
\hline
\end{tabular}

The field experiment on cotton productivity and leaf reddening as influenced by nutrition management for targeted yield was conducted during growing seasons of 2014-15 and 2015-16 at College of Agriculture Farm, Raichur, Karnataka on medium deep black soil under irrigation. Three yield targets $\left(3,4\right.$ and $5 \mathrm{t}$ kapas yield $\left.\mathrm{ha}^{-1}\right)$ based site specific nutrient management (SSNM) along with four leaf reddening management (LRM) treatments (S1 - Vermicompost @ $2.5 \mathrm{t} \mathrm{ha}^{-1}$ in seed line, $\mathrm{S} 2-\mathrm{S} 1+\mathrm{MgSO}_{4} 10 \mathrm{~kg} \mathrm{ha}^{-1}$ in seed line, $\mathrm{S} 3-\mathrm{S} 1+\mathrm{MgSO}_{4} 25 \mathrm{~kg} \mathrm{ha}^{-1}$ in seed line, and S4 - $\mathrm{MgSO}_{4} 25 \mathrm{~kg} \mathrm{ha}^{-1}$ in seed line + foliar nutrition of $1 \% \mathrm{MgSO}_{4}+19: 19: 19+1 \% \mathrm{KNO}_{3}$ trice during flowering, boll development and boll bursting stages) besides recommended control were tested using RCBD. SSNM for $5 \mathrm{t} \mathrm{ha}^{-1}$ yield target and supplementary nutrition of MgSO4 both to soil and to foliage and foliar application of major nutrients (19:19:19 and KNO3) (S4) recorded significantly higher aphids count throughout (3.69, 5.42, 2.87 and 3.3), hopper count (10.7, 15.3, 17.5 and 13.1) and thrips count throughout (14.0, 17.8, 21.1 and 16.6 at 30, 45, 60 and 90 DAS, respectively on pooled basis). The dynamics population was lower with medium to lower yield targets; latter $\left(\mathbf{M}_{1}\right)$ had lower count among all on pooled basis.

\section{Introduction}

Cotton is an important commercial crop unanimously designated as 'king of fibre crops' and is prone to insect pests attack at various stages of crop growth. World total cotton production was recorded 120.97 million bales from the 34.35 million hectares of total 
cultivated area and $767 \mathrm{~kg} /$ hectare productivity in 2012-13 (Anonymous, 2013).

Introduction of synthetic pyrethroids, though brought desirable control of bollworms, resulted in resurgence of sucking pests viz., aphid, Aphis gossypii Glover; leafhopper, Patil et al., 1986). On introduction of Bt cotton, the population and infestation due to major bollworms is now under control. However, year after year, the infestation of sucking pests showed increasing trend. Cultivation of cotton under diversified agro climatic situations makes the crop to suffer a lot by different kinds of pests and diseases. Large area under irrigated situations and extensive application of fertilisers with superior hybrids made the crop easily vulnerable to insect pests.

The major reason for the low productivity in cotton is damage caused by insect pests. In India, as many as 162 species of insect-pests are known to attack cotton from sowing to maturity which cause up to 50-60 per cent loss (Agarwal et al., 1984). Cotton pests can be primarily divided into bollworms and sucking pests. Among sucking pests, aphid, Aphis gossypii (Glover), leafhoppers, Amrasca biguttula biguttula (Ishida), thrips, Thrips tabaci (Lind.) and whitefly, Bemisia tabaci (Genn.) are of major importance.

These sucking pests occur at all the stages of crop growth and responsible for indirect yield losses. A reduction of 22.85 per cent in seed cotton yield due to sucking pests has been reported by Satpute et al., (1990). In the impact assessment of transgenic cottons a little attention has been given on the changing dynamics of sucking pests and other nontarget organisms. With Bt cottons it has been experienced that reduction in usage of insecticides lead to increased population of sucking insect pests (Men et al., 2005). Thus, in Bt cotton era sucking pests are becoming more serious inviting indiscriminate use of pesticides. Hence, the present study was undertaken with objective to know the population dynamics of sucking pest by the site specific nutrient management in Bt cotton.

\section{Materials and Methods}

Experiment was carried out at Agricultural College Farm, University of Agricultural Sciences, Raichur, and Karnataka during growing seasons of 2014-15 and 2015-16 under irrigation. The experiment consisted of three main plot treatments (SSNM based nutrition for 3, 4 and $5 \mathrm{tha}^{-1}$ seed cotton - M13) and four sub plot treatments (nutrient supplementation to manage leaf reddening malady (LRM): S1 - Vermicompost@ $2.5 \mathrm{t}$ $\mathrm{ha}^{-1}$ in seed line, $\mathrm{S} 2-\mathrm{S} 1+\mathrm{MgSO}_{4} 10 \mathrm{~kg} \mathrm{ha}^{-1}$ in seed line, $\mathrm{S} 3-\mathrm{S} 1+\mathrm{MgSO}_{4} 25 \mathrm{~kg} \mathrm{ha}^{-1}$ in seed line and $\mathrm{S} 4-\mathrm{MgSO}_{4} 25 \mathrm{~kg} \mathrm{ha}^{-1}$ in seed line + foliar nutrition of $1 \% \mathrm{MgSO}_{4}+19: 19: 19$ $+1 \% \mathrm{KNO}_{3}$ thrice during flowering, boll development and boll bursting stages) along with recommended fertilizer practice (RDF) as outside control for comparison $(3 \times 4+1)$. For the yield targets fertilizers were applied based on the soil test and crop requirement as per SSNM (IPNI). In control the recommended doses of fertilizers were applied $(150 \mathrm{~N}, 75$ P2O5 and $75 \mathrm{~K} \mathrm{~kg} \mathrm{ha-1).}$

Observations on sucking pests were taken in the leaves at 30, 45, 60 and 90 DAS. Five plants per plot in each treatment were selected and tagged. The number of thrips, leaf hoppers and aphids will be counted on top growing (3, 5 and 7 leaf on the main stem from top) three leaves from each plant.

The mean population per leaf per plant taken from the experiment at different growth stages were subjected to statistical analysis (Gomez and Gomez, 1984) at $\mathrm{P}=0.05$ and means were compared using Duncan's Multiple Range Test (DMRT) using SPSS 16.0 version. Third order interactions were presented and discussed in the article. 


\section{Results and Discussion}

\section{Aphid population (count leaf ${ }^{-1}$ )}

Initially the population was higher up to 45 DAS and thereafter more or less it remained stable, however, variations due to SSNM targets and LRM practices and their interactions were relatively narrow though significant on most of the occasions during both the years and on pooled basis (Table 1). Among SSNM based nutrition, $5 \mathrm{t} \mathrm{ha}^{-1}$ yield target $\left(\mathrm{M}_{3}\right)$ recorded significantly higher aphids count throughout (3.69, 5.42, 2.87 and 3.3 at 30, 45, 60 and 90 DAS, respectively on pooled basis) except at 60 and 90 DAS during 2015-16. The aphid population was lower with medium to lower yield targets; latter $\left(\mathrm{M}_{1}\right)$ had lower count among all (1.46, 1.67, 2.63 and 2.40 at 30, 45, 60 and 90 DAS, respectively on pooled basis) Influence of LRM practices was not astounding too; variations at later stages i.e. at 90 DAS during 2014-15, and at 60 and 90 DAS during 2015-16 and pooled basis were not significant.

In all, $\mathrm{MgSO}_{4}$ to soil and foliage and 19:19:19 and $\mathrm{KNO}_{3}$ to foliage for LRM $\left(\mathrm{S}_{4}\right)$ had consistently higher aphids count (3.35, 4.30, 2.84 and 3.10 at 30, 45, and 60 and 90 DAS, respectively on pooled basis), while vermicompost alone $\left(\mathrm{S}_{1}\right)$ had lower aphids density $(2.17,3.06,2.50$ and 2.60 at 30, 45, 60 and 90 DAS, respectively on pooled basis). Interaction effects also did not vary much except for $5 \mathrm{t} \mathrm{ha}^{-1}$ yield target coupled with $\mathrm{MgSO}_{4}$ to soil and foliage and 19:19:19 and $\mathrm{KNO}_{3}$ to foliage for LRM $\left(\mathrm{M}_{3} \mathrm{~S}_{4}\right)$ which more consistently had higher aphid count (5.27, 6.80, 3.82 and 4.20 at 30, 45, and 60 and 90 DAS, respectively on pooled basis) among all, while others mostly at par among themselves, often particularly at later stages. Interestingly, the recommended control recorded the lowest population $(1.80,3.50,3.0$ and 2.7 at 30,45 , 60 and 90 DAS, respectively on pooled basis).

\section{Leaf hoppers population (count leaf ${ }^{-1}$ )}

Leaf hopper population increased till 60 DAS and thereafter decreased and though significant differences observed due treatments not much could be made out of it except one or two observations (Table 2). Among the SSNM based yield targets, significantly higher hopper count $(10.7,15.3$, 17.5 and 13.1 at 30, 45, 60 and 90 DAS, respectively on pooled basis) was observed with $5 \mathrm{t} \mathrm{ha}^{-1}$ yield target $\left(\mathrm{M}_{3}\right)$ on pooled basis compared to other targets. Application of supplementary nutrition on pooled basis revealed that at 30 DAS highest population (9.0) was recorded with vermicompost + $\mathrm{MgSO}_{4} 10 \mathrm{~kg} \mathrm{ha}^{-1}$ in seed line $\left(\mathrm{S}_{2}\right)$ and lowest (6.0) was recorded with vermicompost application $\left(S_{1}\right)$. Application of vermicompost along with $\mathrm{MgSO}_{4} 25 \mathrm{~kg} \mathrm{ha}^{-1}$ in seed line $\left(\mathrm{S}_{3}\right)$ and $\mathrm{MgSO}_{4} 25 \mathrm{~kg} \mathrm{ha}^{-1}$ in seed line + foliar nutrition of 1\% each of $\mathrm{MgSO}_{4}, 19: 19: 19$ and $1 \% \mathrm{KNO}_{3}$ (thrice each) were on par with each other (7.6 and 7.6).

Among the treatment combinations, $5 \mathrm{t} \mathrm{ha}^{-1}$ yield target irrespective LRM interventions were mostly at par and recorded higher count, while it decreased with lower yield target and with vermicompost. Hopper count with control $(8.7,9.2,9.1$ and 7.8 at 30, 45, 60 and 90 DAS, respectively on pooled basis) was somewhere in between or on lower side during 30 and 60 DAS but was significantly lower than those of any of the treatment combinations thereafter.

\section{Thrips population (count leaf ${ }^{-1}$ )}

Thrips population increased from the beginning till 60 DAS and declined thereafter. SSNM based nutrition for targeted yield and supplementary nutrition for leaf reddening control and their interactions resulted in significant variation in thrips population at all the stages of observation (Table 2). 
Table.1 Soil test value, ratings, and nutrient requirement to achieve the target and adjusted nutrients for the I experiment during 2014-15 and 2015-16

\begin{tabular}{|c|c|c|c|c|}
\hline \multirow{2}{*}{$\begin{array}{l}\text { Yield } \\
\text { Targets }\end{array}$} & \multicolumn{2}{|c|}{ Soil test value $\left(\mathrm{N}: \mathrm{P}_{2} \mathrm{O}_{5}: \mathrm{K}_{2} \mathrm{O} \mathrm{kg} \mathrm{ha}^{-1}\right)$} & \multirow{2}{*}{$\begin{array}{l}\text { Nutrient requirement } \\
\left(\mathrm{N}: \mathrm{P}_{2} \mathrm{O}_{5}: \mathrm{K}_{2} \mathrm{O} \mathrm{kg} \mathrm{ha}^{-1}\right)\end{array}$} & \multirow{2}{*}{$\begin{array}{c}\text { Final applied } \\
\left(\mathrm{N}: \mathrm{P}_{2} \mathrm{O}_{5}: \mathrm{K}_{2} \mathrm{O} \mathrm{kg} \mathrm{ha}^{-1}\right)\end{array}$} \\
\hline & 2014-15 & 2015-16 & & \\
\hline $3 \mathrm{t} \mathrm{ha}^{-1}$ & $168: 72: 184$ & 198:74:208 & 192:84:114 & $240: 63: 114$ \\
\hline $4 \mathrm{tha}^{-1}$ & $168: 72: 184$ & 198:74:208 & $256: 112: 152$ & $316: 84: 152$ \\
\hline $5 \mathrm{tha}^{-1}$ & 168:72:184 & 198:74:208 & 320:140:190 & $400: 105: 190$ \\
\hline
\end{tabular}

Table.2 Sucking pest population per leaf of cotton at various stages as influenced by SSNM based yield targets and nutrition for leaf reddening management (S) (pooled data of two years)

\begin{tabular}{|c|c|c|c|c|c|c|c|c|c|c|c|c|}
\hline \multirow[t]{2}{*}{ Treatments } & \multicolumn{4}{|c|}{ Aphids populations } & \multicolumn{4}{|c|}{ Leaf hoppers populations } & \multicolumn{4}{|c|}{ Thrips populations } \\
\hline & $\begin{array}{c}30 \\
\text { DAS }\end{array}$ & 45 DAS & $\begin{array}{c}60 \\
\text { DAS }\end{array}$ & $\begin{array}{c}90 \\
\text { DAS }\end{array}$ & $\begin{array}{c}30 \\
\text { DAS }\end{array}$ & $\begin{array}{c}45 \\
\text { DAS }\end{array}$ & $\begin{array}{c}60 \\
\text { DAS }\end{array}$ & $\begin{array}{c}90 \\
\text { DAS }\end{array}$ & $\begin{array}{c}30 \\
\text { DAS }\end{array}$ & $\begin{array}{c}45 \\
\text { DAS }\end{array}$ & $\begin{array}{c}60 \\
\text { DAS }\end{array}$ & $\begin{array}{c}90 \\
\text { DAS }\end{array}$ \\
\hline \multicolumn{13}{|l|}{ Main plots } \\
\hline$M_{1}$ & $1.46^{\mathrm{c}}$ & $1.67^{\mathrm{c}}$ & $2.63^{\mathrm{a}}$ & $2.4^{\mathrm{b}}$ & $6.7^{\mathrm{b}}$ & $9.5^{\mathrm{c}}$ & $12.0^{\mathrm{b}}$ & $9.1^{\mathrm{b}}$ & $9.6^{c}$ & $12.7^{\mathrm{c}}$ & $16.5^{\mathrm{b}}$ & $11.8^{\mathrm{b}}$ \\
\hline $\mathbf{M}_{2}$ & $2.43^{b}$ & $3.09^{b}$ & $2.74^{\mathrm{a}}$ & $2.9^{\text {ba }}$ & $5.4^{\mathrm{b}}$ & $12.2^{\mathrm{b}}$ & $12.9^{b}$ & $9.7^{\mathrm{b}}$ & $11.0^{\mathrm{b}}$ & $13.5^{\mathrm{b}}$ & $16.1^{\mathrm{b}}$ & $12.5^{\mathrm{b}}$ \\
\hline $\mathbf{M}_{3}$ & $3.69^{\mathrm{a}}$ & $5.42^{\mathrm{a}}$ & $2.87^{\mathrm{a}}$ & $3.3^{\mathrm{a}}$ & $10.7^{\mathrm{a}}$ & $15.3^{\mathrm{a}}$ & $17.5^{\mathrm{a}}$ & $13.1^{\mathrm{a}}$ & $14.0^{\mathrm{a}}$ & $17.8^{\mathrm{a}}$ & $21.1^{\mathrm{a}}$ & $16.6^{\mathrm{a}}$ \\
\hline S.Em \pm & 0.04 & 0.11 & 0.23 & 0.50 & 0.40 & 0.30 & 0.30 & 0.50 & 0.10 & 0.20 & 0.30 & 0.20 \\
\hline \multicolumn{13}{|l|}{ Sub plots } \\
\hline$S_{1}$ & $2.17^{\mathrm{b}}$ & $3.06^{\mathrm{b}}$ & $2.50^{\mathrm{a}}$ & $2.6^{\mathrm{a}}$ & $6.0^{\mathrm{c}}$ & $11.7^{\mathrm{b}}$ & $15.0^{\mathrm{a}}$ & $9.8^{\mathrm{b}}$ & $10.6^{\mathrm{b}}$ & $14.6^{\mathrm{a}}$ & $17.9^{\mathrm{a}}$ & $13.5^{\mathrm{a}}$ \\
\hline$\underline{S_{2}}$ & $2.13^{b}$ & $2.89^{\mathrm{b}}$ & $3.14^{\mathrm{a}}$ & $3.1^{\mathrm{a}}$ & $9.0^{\mathrm{a}}$ & $13.3^{\mathrm{a}}$ & $14.4^{\mathrm{ba}}$ & $11.6^{\mathrm{ba}}$ & $11.1^{\mathrm{ba}}$ & $13.1^{\mathrm{b}}$ & $15.9^{\mathrm{b}}$ & $12.3^{\mathrm{b}}$ \\
\hline$S_{3}$ & $2.46^{\mathrm{b}}$ & $3.33^{\mathrm{b}}$ & $2.50^{\mathrm{a}}$ & $2.6^{\mathrm{a}}$ & $7.6^{b}$ & $12.6^{\mathrm{ba}}$ & $13.7^{\text {ba }}$ & $10.8^{\mathrm{ba}}$ & $12.3^{\mathrm{a}}$ & $15.6^{\mathrm{a}}$ & $18.7^{\mathrm{a}}$ & $14.5^{\mathrm{a}}$ \\
\hline$\underline{S_{4}}$ & $3.35^{\mathrm{a}}$ & $4.30^{\mathrm{a}}$ & $2.84^{\mathrm{a}}$ & $3.1^{\mathrm{a}}$ & $7.6^{\mathrm{b}}$ & $11.7^{b}$ & $13.4^{\mathrm{b}}$ & $10.3^{\text {ba }}$ & $12.2^{\mathrm{a}}$ & $15.3^{\mathrm{a}}$ & $18.9^{\mathrm{a}}$ & $14.4^{\mathrm{a}}$ \\
\hline S.Em \pm & 0.07 & 0.11 & 0.09 & 0.20 & 0.10 & 0.20 & 0.30 & 0.30 & 0.20 & 0.30 & 0.30 & 0.40 \\
\hline \multicolumn{13}{|l|}{$\mathbf{M} \times \mathrm{S}$} \\
\hline $\mathrm{M}_{1} \mathrm{~S}_{1}$ & $1.42^{\mathrm{c}}$ & $1.64^{\mathrm{g}}$ & $2.88^{\text {ba }}$ & $2.5^{\mathrm{b}}$ & $4.8^{\mathrm{de}}$ & $8.7^{\mathrm{f}}$ & $12.1^{\mathrm{cb}}$ & $8.0^{c}$ & $7.4^{\mathrm{e}}$ & $12.1^{\mathrm{cb}}$ & $15.0^{\text {def }}$ & $11.0^{\mathrm{e}}$ \\
\hline $\mathrm{M}_{1} \mathrm{~S}_{2}$ & $1.45^{\mathrm{c}}$ & $1.63^{\mathrm{g}}$ & $3.75^{\mathrm{a}}$ & $3.3^{\mathrm{b}}$ & $8.4^{\mathrm{c}}$ & $10.5^{\text {dfe }}$ & $13.2^{\mathrm{cb}}$ & $10.4^{\mathrm{bc}}$ & $9.5^{\mathrm{ecd}}$ & $11.5^{\mathrm{c}}$ & $14.7^{\mathrm{ef}}$ & $10.7^{\mathrm{e}}$ \\
\hline $\mathbf{M}_{1} \mathbf{S}_{3}$ & $1.48^{\mathrm{c}}$ & $1.69^{\mathrm{g}}$ & $1.72^{\mathrm{b}}$ & $1.6^{\mathrm{b}}$ & $6.6^{\mathrm{dce}}$ & $9.7^{\mathrm{fe}}$ & $11.6^{\mathrm{cb}}$ & $9.1^{c}$ & $10.2^{\text {cd }}$ & $14.4^{\mathrm{cb}}$ & $16.7^{\mathrm{de}}$ & $13.2^{\mathrm{dc}}$ \\
\hline $\mathbf{M}_{1} \mathbf{S}_{4}$ & $1.50^{\mathrm{c}}$ & $1.74^{\mathrm{g}}$ & $2.18^{\text {ba }}$ & $2.0^{\mathrm{b}}$ & $6.9^{\mathrm{dc}}$ & $9.2^{\mathrm{f}}$ & $11.1^{\mathrm{c}}$ & $8.9^{c}$ & $11.4^{\mathrm{cd}}$ & $12.8^{\mathrm{cb}}$ & $19.5^{\mathrm{bc}}$ & $12.4^{\text {dce }}$ \\
\hline $\mathbf{M}_{2} \mathbf{S}_{1}$ & $1.72^{\mathrm{c}}$ & $2.27^{\mathrm{gf}}$ & $1.97^{\mathrm{b}}$ & $2.1^{\mathrm{b}}$ & $4.3^{\mathrm{e}}$ & $12.0^{\mathrm{dc}}$ & $14.1^{\mathrm{b}}$ & $9.2^{\mathrm{c}}$ & $9.3^{\text {ed }}$ & $14.0^{\mathrm{cb}}$ & $17.4^{\mathrm{cc}}$ & $12.7^{\text {dce }}$ \\
\hline $\mathbf{M}_{2} \mathbf{S}_{2}$ & $2.32^{\mathrm{cb}}$ & $3.16^{\operatorname{det}}$ & $3.22^{\mathrm{ba}}$ & $3.4^{\mathrm{b}}$ & $7.0^{\mathrm{dc}}$ & $13.0^{\mathrm{bc}}$ & $11.9^{\mathrm{cb}}$ & $10.3^{\mathrm{bc}}$ & $12.2^{\mathrm{bc}}$ & $12.8^{\mathrm{cb}}$ & $13.7^{\mathrm{t}}$ & $12.2^{\text {dce }}$ \\
\hline $\mathbf{M}_{2} \mathbf{S}_{3}$ & $2.40^{\mathrm{cb}}$ & $2.58^{\mathrm{gef}}$ & $3.25^{\text {ba }}$ & $3.2^{\mathrm{b}}$ & $5.4^{\mathrm{de}}$ & $12.3^{\mathrm{dc}}$ & $13.1^{\mathrm{cb}}$ & $10.0^{\mathrm{bc}}$ & $11.6^{\mathrm{bcd}}$ & $12.2^{\mathrm{cb}}$ & $16.5^{\mathrm{de}}$ & $11.6^{\mathrm{de}}$ \\
\hline $\mathbf{M}_{2} \mathbf{S}_{4}$ & $3.28^{\mathrm{b}}$ & $4.36^{\mathrm{dc}}$ & $2.53^{\text {ba }}$ & $3.0^{6}$ & $4.8^{\mathrm{de}}$ & $11.7^{\mathrm{dce}}$ & $12.6^{\mathrm{cb}}$ & $9.4^{c}$ & $11.1^{\mathrm{cd}}$ & $15.1^{\mathrm{b}}-\mathrm{c} \cdot \mathrm{c}$ & $16.6^{\mathrm{de}}$ & $13.8^{\mathrm{c}}$ \\
\hline $\mathbf{M}_{3} \mathbf{S}_{1}$ & $3.38^{\mathrm{b}}$ & $5.27^{\mathrm{bc}}$ & $2.67^{\text {ba }}$ & $3.3^{\mathrm{b}}$ & $9.0^{\mathrm{bc}}$ & $14.5^{\mathrm{ba}}$ & $18.6^{\mathrm{a}}$ & $12.4^{\mathrm{ba}}$ & $15.0^{\mathrm{a}}$ & $17.8^{\mathrm{a}}$ & $21.4^{\mathrm{bc}}$ & $16.8^{\mathrm{b}}$ \\
\hline $\mathbf{M}_{3} \mathbf{S}_{2}$ & $2.62^{\mathrm{c}}$ & $3.87^{\mathrm{de}}$ & $2.45^{\text {ba }}$ & $2.7^{\mathrm{b}}$ & $11.7^{\mathrm{a}}$ & $16.5^{\mathrm{a}}$ & $18.1^{\mathrm{a}}$ & $14.1^{\mathrm{a}}$ & $11.8^{\text {bcd }}$ & $15.0^{\mathrm{b}}$ & $19.3^{\mathrm{bc}}$ & $14.0^{c}$ \\
\hline $\mathrm{M}_{3} \mathrm{~S}_{3}$ & $3.48^{\mathrm{b}}$ & $5.73^{\text {ba }}$ & $2.53^{\mathrm{ba}}$ & $3.0^{\mathrm{b}}$ & $11.0^{\mathrm{ba}}$ & $15.8^{\mathrm{a}}$ & $16.5^{\mathrm{a}}$ & $13.3^{\mathrm{a}}$ & $15.3^{\mathrm{a}}$ & $20.4^{\mathrm{a}}$ & $23.0^{\mathrm{a}}$ & $18.8^{\mathrm{a}}$ \\
\hline $\mathbf{M}_{3} \mathbf{S}_{4}$ & $5.27^{\mathrm{a}}$ & $6.80^{\mathrm{a}}$ & $3.82^{\mathrm{a}}$ & $4.2^{\mathrm{a}}$ & $11.1^{\mathrm{ba}}$ & $14.4^{\mathrm{ba}}$ & $16.7^{\mathrm{a}}$ & $12.8^{\text {ba }}$ & $14.1^{\text {ba }}$ & $18.0^{\mathrm{a}}$ & $20.6^{\mathrm{b}}$ & $16.9^{\text {ba }}$ \\
\hline S.Em \pm & 0.12 & 0.20 & 0.27 & 0.5 & 0.4 & 0.4 & 0.5 & 0.7 & 0.3 & 0.5 & 0.6 & 0.7 \\
\hline Control & 1.8 & 3.5 & 3.0 & 2.7 & 8.7 & 9.2 & 9.1 & 7.8 & 7.1 & 11.6 & 16.1 & 16.8 \\
\hline S.Em \pm & 0.16 & 0.23 & 0.17 & 0.39 & 0.16 & 0.87 & 0.75 & 0.97 & 0.33 & 0.64 & 0.32 & 0.69 \\
\hline C.D. 0.05 & 0.42 & 0.70 & 0.49 & 1.1 & 0.5 & 2.5 & 1.6 & 2.8 & 0.9 & 1.9 & 0.9 & 2.0 \\
\hline
\end{tabular}

*means with same letters do not differ significantly under DMRT

Note: DAS - Days after sowing, SSNM- Site Specific Nutrient Management

Main treatments: Yield Target (M)

$\mathrm{M}_{1}$ - SSNM for targeted yield of 3 tha $^{-1}$

$M_{2}$ - SSNM for targeted yield of 4 tha $^{-1}$

$\mathrm{M}_{3}$ - SSNM for targeted yield of 5 tha $^{-1}$

Control-RDF with recommended practice
Sub treatments: Leaf reddening management (S)

$\mathbf{S}_{1^{-}}$Vermicompost @ 2.5 tha $^{-1}$ in seed line

$\mathbf{S}_{2^{-}} \mathrm{S}_{1}+\mathrm{MgSO}_{4} 10 \mathrm{kgha}^{-1}$ in seed line

$\mathbf{S}_{3}-\mathrm{S}_{1}+\mathrm{MgSO}_{4} 25 \mathrm{kgha}^{-1}$ in seed line

$\mathbf{S}_{4^{-}}-\mathrm{MgSO}_{4} 25 \mathrm{~kg} \mathrm{ha}^{-1}$ in seed line + foliar nutrition of $1 \%$

$\mathrm{MgSO}_{4}+19: 19: 19+1 \% \mathrm{KNO}_{3}$ (thrice each) 
Table.3 Sucking pest population per leaf (Transformed $\sqrt{ } \mathrm{x}+0.5$ ) of cotton at various stages as influenced by SSNM based yield targets and nutrition for leaf reddening management (S)

(pooled data of two years)

\begin{tabular}{|c|c|c|c|c|c|c|c|c|c|c|c|c|}
\hline \multirow[t]{2}{*}{ Treatments } & \multicolumn{4}{|c|}{ Aphids populations } & \multicolumn{4}{|c|}{ Leaf hoppers populations } & \multicolumn{4}{|c|}{ Thrips populations } \\
\hline & $\begin{array}{c}30 \\
\text { DAS }\end{array}$ & $\begin{array}{c}45 \\
\text { DAS }\end{array}$ & $\begin{array}{c}60 \\
\text { DAS }\end{array}$ & $\begin{array}{c}90 \\
\text { DAS }\end{array}$ & $\begin{array}{c}30 \\
\text { DAS }\end{array}$ & 45 DAS & $\begin{array}{c}60 \\
\text { DAS }\end{array}$ & $\begin{array}{c}90 \\
\text { DAS }\end{array}$ & $\begin{array}{c}30 \\
\text { DAS }\end{array}$ & $\begin{array}{c}45 \\
\text { DAS }\end{array}$ & $\begin{array}{c}60 \\
\text { DAS }\end{array}$ & $\begin{array}{c}90 \\
\text { DAS }\end{array}$ \\
\hline \multicolumn{13}{|l|}{ Main plots } \\
\hline $\mathbf{M}_{1}$ & $1.61^{\mathrm{c}}$ & $1.67^{c}$ & $1.85 \mathrm{a}$ & $1.62^{b}$ & $2.80^{\mathrm{b}}$ & $3.48^{\mathrm{c}}$ & $3.40^{\mathrm{b}}$ & $3.15^{\mathrm{b}}$ & $3.19^{c}$ & $3.53^{\mathrm{c}}$ & $4.23^{\mathrm{b}}$ & $3.36^{\mathrm{b}}$ \\
\hline$\overline{M_{2}}$ & $2.07^{\mathrm{b}}$ & $2.20^{\mathrm{b}}$ & $1.95 \mathrm{a}$ & $2.07^{\mathrm{ba}}$ & $2.67^{\mathrm{b}}$ & $4.00^{\mathrm{b}}$ & $3.64^{\mathrm{b}}$ & $3.34^{\mathrm{b}}$ & $3.43^{\mathrm{b}}$ & $3.72^{\mathrm{b}}$ & $4.28^{\mathrm{b}}$ & $3.46^{\mathrm{b}}$ \\
\hline $\mathrm{M}_{3}$ & $2.53^{\mathrm{a}}$ & $2.95^{\mathrm{a}}$ & $2.18 \mathrm{a}$ & $2.24^{\mathrm{a}}$ & $3.79^{\mathrm{a}}$ & $4.45^{\mathrm{a}}$ & $4.12^{\mathrm{a}}$ & $3.39^{\mathrm{a}}$ & $3.96^{\mathrm{a}}$ & $4.32^{\mathrm{a}}$ & $4.94^{\mathrm{a}}$ & $3.98^{\mathrm{a}}$ \\
\hline S.Emt & 0.13 & 0.15 & 0.24 & 0.24 & 0.21 & 0.12 & 0.07 & 0.25 & 0.08 & 0.18 & 0.10 & 0.03 \\
\hline \multicolumn{13}{|l|}{ Sub plots } \\
\hline$S_{1}$ & $1.96^{\mathrm{b}}$ & $2.19^{\mathrm{b}}$ & $1.93^{\mathrm{a}}$ & $1.91^{\mathrm{a}}$ & $2.79^{c}$ & $3.89^{b}$ & $3.83^{\mathrm{a}}$ & $3.15^{\mathrm{b}}$ & $3.36^{\mathrm{b}}$ & $3.86^{\mathrm{a}}$ & $4.49^{\mathrm{a}}$ & $3.58^{\mathrm{a}}$ \\
\hline$S_{2}$ & $1.95^{\mathrm{b}}$ & $2.16^{b}$ & $2.11^{\mathrm{a}}$ & $2.05^{\mathrm{a}}$ & $3.37^{\mathrm{a}}$ & $4.12^{\mathrm{a}}$ & $3.75^{\mathrm{ab}}$ & $3.45^{\mathrm{ba}}$ & $3.48^{\mathrm{ba}}$ & $3.66^{\mathrm{b}}$ & $4.24^{\mathrm{b}}$ & $3.43^{\mathrm{b}}$ \\
\hline$S_{3}$ & $2.06^{\mathrm{b}}$ & $2.25^{b}$ & $1.92^{\mathrm{a}}$ & $1.91^{\mathrm{a}}$ & $3.11^{\mathrm{b}}$ & $4.01^{\mathrm{ba}}$ & $3.67^{\mathrm{ab}}$ & $3.32^{\mathrm{ba}}$ & $3.64^{\mathrm{a}}$ & $3.97^{\mathrm{a}}$ & $4.58^{\mathrm{a}}$ & $3.70^{\mathrm{a}}$ \\
\hline$S_{4}$ & $2.30^{\mathrm{a}}$ & $2.49^{\mathrm{a}}$ & $2.01^{\mathrm{a}}$ & $2.03^{\mathrm{a}}$ & $3.08^{b}$ & $3.89^{b}$ & $3.62^{b}$ & $3.24^{\mathrm{ba}}$ & $3.62^{\mathrm{a}}$ & $3.94^{\mathrm{a}}$ & $4.61^{\mathrm{a}}$ & $3.70^{\mathrm{a}}$ \\
\hline S.Em \pm & 0.03 & 0.05 & 0.06 & 0.07 & 0.09 & 0.03 & 0.04 & 0.06 & 0.03 & 0.03 & 0.03 & 0.05 \\
\hline \multicolumn{13}{|l|}{$\mathrm{MxS}$} \\
\hline $\mathrm{M}_{1} \mathrm{~S}_{1}$ & $1.59^{\mathrm{c}}$ & $1.66 \mathrm{~g}$ & $1.95^{\mathrm{ba}}$ & $1.67^{b}$ & $2.42^{\mathrm{de}}$ & $3.35^{\mathrm{f}}$ & $3.42^{\mathrm{cb}}$ & $2.95^{\mathrm{c}}$ & $2.83^{\mathrm{e}}$ & $3.45^{\mathrm{cb}}$ & $4.05^{\mathrm{def}}$ & $3.24^{\mathrm{e}}$ \\
\hline $\mathbf{M}_{1} \mathbf{S}_{2}$ & $1.60^{c}$ & $1.66 \mathrm{~g}$ & $2.18^{\mathrm{a}}$ & $1.90^{\mathrm{b}}$ & $3.13^{c}$ & $3.64^{\mathrm{dfe}}$ & $3.56^{\mathrm{cb}}$ & $3.37^{\mathrm{bc}}$ & $3.17^{\text {ecd }}$ & $3.36^{\mathrm{c}}$ & $4.02^{\mathrm{ef}}$ & $3.21^{\mathrm{e}}$ \\
\hline $\mathbf{M}_{1} \mathbf{S}_{3}$ & $1.61^{\mathrm{c}}$ & $1.67 \mathrm{~g}$ & $1.57^{b}$ & $1.41^{b}$ & $2.81^{\mathrm{dce}}$ & $3.51^{\mathrm{fe}}$ & $3.34^{\mathrm{cb}}$ & $3.16^{\mathrm{c}}$ & $3.29^{\mathrm{cd}}$ & $3.75^{\mathrm{cb}}$ & $4.26^{\mathrm{de}}$ & $3.55^{\mathrm{dc}}$ \\
\hline $\mathbf{M}_{1} \mathbf{S}_{4}$ & $1.62^{\mathrm{c}}$ & $1.69 \mathrm{~g}$ & 1.71 & $1.51^{\mathrm{b}}$ & $2.86^{\mathrm{dc}}$ & $3.42^{\mathrm{f}}$ & $3.26^{\mathrm{c}}$ & $3.12^{\mathrm{c}}$ & $3.48^{\mathrm{cd}}$ & $3.54^{\mathrm{cb}}$ & $4.59^{\mathrm{bc}}$ & $3.45^{\mathrm{dce}}$ \\
\hline $\mathrm{M}_{2} \mathrm{~S}_{1}$ & $1.83^{c}$ & $1.97^{\mathrm{gf}}$ & $1.71^{\mathrm{b}}$ & $1.83^{b}$ & $2.44^{\mathrm{e}}$ & $3.97^{\mathrm{dc}}$ & $3.81^{\mathrm{b}}$ & $3.24^{\mathrm{c}}$ & $3.17^{\text {ed }}$ & $3.80^{\mathrm{cb}}$ & $4.45^{\mathrm{dc}}$ & $3.48^{\mathrm{dce}}$ \\
\hline$\overline{\mathbf{M}_{2} \mathrm{~S}_{2}}$ & $2.04^{\mathrm{cb}}$ & $2.25^{\mathrm{def}}$ & 2.10 & $2.20^{\mathrm{b}}$ & $3.04^{\mathrm{dc}}$ & $4.12^{\mathrm{bc}}$ & $3.50^{\mathrm{cb}}$ & $3.46^{\mathrm{bc}}$ & $3.60^{b c}$ & $3.63^{\mathrm{cb}}$ & $3.98^{f}$ & $3.42^{\mathrm{dce}}$ \\
\hline $\mathrm{M}_{2} \mathrm{~S}_{3}$ & $2.07^{\mathrm{cb}}$ & $2.04^{\mathrm{gef}}$ & 2.11 & $2.16^{\mathrm{b}}$ & $2.68^{\mathrm{de}}$ & $4.02^{\mathrm{dc}}$ & $3.66^{\mathrm{cb}}$ & $3.38^{\mathrm{bc}}$ & $3.51^{\mathrm{bcd}}$ & $3.53^{\mathrm{cd}}$ & $4.34^{\mathrm{de}}$ & $3.32^{\mathrm{de}}$ \\
\hline $\mathrm{M}_{2} \mathrm{~S}_{4}$ & $2.34^{\mathrm{b}}$ & $2.55^{\mathrm{dc}}$ & 1.87 & $2.08^{b}$ & $2.51^{\mathrm{de}}$ & $3.92^{\text {dce }}$ & $3.58^{\mathrm{cb}}$ & $3.26^{\mathrm{c}}$ & $3.43^{\mathrm{cd}}$ & $3.92^{\mathrm{b}}$ & $4.35^{\mathrm{de}}$ & $3.63^{c}$ \\
\hline $\mathbf{M}_{3} \mathbf{S}_{1}$ & $2.47^{\mathrm{b}}$ & $2.94^{\mathrm{bc}}$ & 2.13 & $2.24^{\mathrm{b}}$ & $3.52^{\mathrm{bc}}$ & $4.35^{\mathrm{ba}}$ & $4.25^{\mathrm{a}}$ & $3.27^{\mathrm{ba}}$ & $4.09^{\mathrm{a}}$ & $4.33^{\mathrm{a}}$ & $4.98^{\mathrm{bc}}$ & $4.01^{\mathrm{b}}$ \\
\hline $\mathbf{M}_{3} \mathbf{S}_{2}$ & $2.22^{\mathrm{cb}}$ & 2.57 & 2.05 & $2.07^{b}$ & $3.94^{\mathrm{a}}$ & $4.60^{a}$ & $4.19^{\mathrm{a}}$ & $3.52^{\mathrm{a}}$ & $3.66^{\mathrm{bcd}}$ & $3.98^{\mathrm{b}}$ & $4.74^{\mathrm{bc}}$ & $3.65^{\mathrm{c}}$ \\
\hline $\mathbf{M}_{3} \mathbf{S}_{3}$ & $2.49^{b}$ & 3.03 & 2.07 & $2.14^{b}$ & $3.84^{\text {ba }}$ & $4.5^{\mathrm{a}}$ & $4.01^{\mathrm{a}}$ & $3.41^{\mathrm{a}}$ & $4.13^{\mathrm{a}}$ & $4.62^{\mathrm{a}}$ & $5.14^{\mathrm{a}}$ & $4.24^{\mathrm{a}}$ \\
\hline $\mathrm{M}_{3} \mathrm{~S}_{4}$ & $2.94^{\mathrm{a}}$ & $3.25^{\mathrm{a}}$ & $2.46^{\mathrm{a}}$ & $2.49^{\mathrm{a}}$ & $3.86^{\mathrm{ba}}$ & $4.33^{\mathrm{ba}}$ & $4.02^{\mathrm{a}}$ & $3.35^{\mathrm{ba}}$ & $3.97^{\mathrm{ba}}$ & $4.36^{\mathrm{a}}$ & $4.88^{\mathrm{b}}$ & $4.02^{\mathrm{ba}}$ \\
\hline S.Em \pm & 0.14 & 0.26 & 0.25 & 0.24 & 0.30 & 0.11 & 0.09 & 0.26 & 0.09 & 0.11 & 0.11 & 0.09 \\
\hline Control & 1.71 & 2.15 & 1.87 & 1.66 & 2.95 & 3.25 & 2.25 & 2.36 & 3.15 & 2.91 & 3.86 & 4.02 \\
\hline S.Em \pm & 0.13 & 0.14 & 0.18 & 0.23 & 0.18 & 0.17 & 0.19 & 0.23 & 0.08 & 0.15 & 0.20 & 0.09 \\
\hline C.D. 0.05 & 0.37 & 0.40 & 0.51 & 0.67 & 0.52 & 0.50 & 0.55 & 0.66 & 0.23 & 0.44 & 0.58 & 0.26 \\
\hline
\end{tabular}

*means with same letters do not differ significantly under DMRT

Note: DAS - Days after sowing, SSNM- Site Specific Nutrient Management

Main treatments: Yield Target (M)

$\mathrm{M}_{1}$ - SSNM for targeted yield of 3 tha $^{-1}$

$\mathrm{M}_{2}$ - SSNM for targeted yield of 4 tha $^{-1}$

$\mathrm{M}_{3^{-}}$SSNM for targeted yield of 5 tha $^{-1}$

Control-RDF with recommended practice
Sub treatments: Leaf reddening management (S)

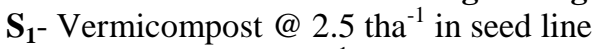

$\mathbf{S}_{2^{-}} \mathrm{S}_{1}+\mathrm{MgSO}_{4} 10 \mathrm{kgha}^{-1}$ in seed line

$\mathrm{S}_{3}-\mathrm{S}_{1}+\mathrm{MgSO}_{4} 25 \mathrm{kgha}^{-1}$ in seed line

$\mathbf{S}_{4^{-}}-\mathrm{MgSO}_{4} 25 \mathrm{~kg} \mathrm{ha}^{-1}$ in seed line + foliar nutrition of $1 \%$

$\mathrm{MgSO}_{4}+19: 19: 19+1 \% \mathrm{KNO}_{3}$ (thrice each
Among the SSNM based yield targets, $5 \mathrm{t} \mathrm{ha}^{-1}$ yield target $\left(\mathrm{M}_{3}\right)$ had significantly higher population throughout (14.0, 17.8, 21.1 and 16.6 at 30, 45, 60 and 90 DAS, respectively on pooled basis) while 4 of $3 \mathrm{t} \mathrm{ha}^{-1}$ were comparable except at 30 DAS and had significantly lower thrips count. Among the supplementary nutrition practices for LRM, vermicompost $+\mathrm{MgSO}_{4}$ to soil at $25 \mathrm{~kg} \mathrm{ha}^{-1}$ $\left(\mathrm{S}_{3}\right)$ and $\mathrm{MgSO}_{4} 25 \mathrm{~kg} \mathrm{ha}^{-1}$ in seed line + foliar nutrition of 1\% each of $\mathrm{MgSO}_{4}, 19: 19: 19$ and $\mathrm{KNO}_{3}$ (thrice each) $\left(\mathrm{S}_{4}\right)$ had higher and comparable thrips count $(12.3,15.6,18.7$ and 14.5 and 12.2, 15.3, 1839 and 14.4, respectively 
with $S_{3}$ and $S_{4}$, at 30, 45, 60 and 90 DAS, respectively) and so was $S_{1}$ from 45-90 DAS.

Interaction effect though revealed significant differences, $5 \mathrm{t} \mathrm{ha}^{-1}$ coupled with vermicompost $+\mathrm{MgSO}_{4}$ to soil at $25 \mathrm{~kg} \mathrm{ha}^{-1}\left(\mathrm{M}_{3} \mathrm{~S}_{3}\right)$ had higher thrips count consistently $(15.3,20.4,23.0$ and 18.8 at 30, 45, 60 and 90 DAS, respectively on pooled basis); $M_{3} S_{4}$ and $M_{3} S_{1}$ were at par at times.

Thrips population decreased with lowering of yield target and different LRM practices were overlapping in their influences.

While, control with recommended practices had significantly lower $(7.1,11.6,16.1$ and 16.8 at 30, 45, 60 and $90 \mathrm{DAS}$, respectively on pooled basis) thrips count than former treatment combinations $\left(\mathrm{M}_{3} \mathrm{~S}_{3}, \mathrm{M}_{3} \mathrm{~S}_{4}\right.$ and $\left.\mathrm{M}_{3} \mathrm{~S}_{1}\right)$.

Sucking pest population was higher up to 45 DAS and thereafter more or less it remained stable (Table 2 and 3 ).

In the present investigation, the highest aphid (5.27, 6.80, 3.82 and 4.20 at 30, 45, 60 and 90 DAS, respectively), leaf hoppers (10.7, 15.3, 17.5 and 13.1 at 30, 45, 60 and 90 DAS, respectively) and thrips (15.3, 20.4, 23.0 and 18.8 at $30,45,60$ and 90 DAS, respectively) count were recorded with the $5 \mathrm{t} \mathrm{ha}^{-1}$ yield target in combination with application of $\mathrm{MgSO}_{4} 25 \mathrm{~kg} \mathrm{ha}^{-1}$ to soil and foliar nutrition of $1 \%$ each of $\mathrm{MgSO}_{4}, 19: 19: 19$ and $\mathrm{KNO}_{3}$ $\left(\mathrm{M}_{3} \mathrm{~S}_{4}\right)$.

This is quite common with nitrogen, as higher dose of $\mathrm{N}$ fertilizer would lead to lush green and succulent plants that attract more of sucking pests than a nutritionally starved crop. The former treatment $\left(\mathrm{M}_{3} \mathrm{~S}_{4}\right)$ had higher $\mathrm{N}$ uptake consequently higher leaf $\mathrm{N}$ content, besides higher dry matter (leaf DM and TDM) and leaf canopy ( $>$ LA and LAI). It was more green ( $>$ 'a', 'b' and total chlorophyll, SPAD and NDVI, and lower leaf anthocyanin and LRI) also in comparison to other treatment combinations and control.

Another reason attributed by Ahmed et al., (2007) was that later doses of fertilizers induce more pest attack due to profuse and succulent plant growth, besides such a growth would also come in the way of efficient coverage of pesticide spray.

\section{References}

Agarwal, R. A., Gupta, G. P. and Garg, D. O., 1984, Cotton pest management in India. Res. Publn., Azadnagar, Delhi, pp. 1-19.

Ahmed, S., Habibulla, Shahzad Sabir and Musthaq Ali., 2007, Effect of different doses of nitrogen fertilizer on sucking pests of cotton (Gossypium hirsutum L.). J. Agric. Res., 45(1): 37-43.

Men, X., Ge, F., Edwards, C. A. and Yardim, E. N., 2005. The influence of pesticide applications on Helicoverpa armigera and sucking pests in transgenic Bt cotton and non-transgenic cotton in China. Crop Prot., 24: 319-324.

Patil, B.K., Rote, N.B. and Mehta, N.P. (1986). Resurgence of sucking pests by the use of synthetic pyrethroids on cotton. In: Proc. Nat. Symp. on resurgence of sucking pests, Tamil Nadu Agricultural University, Coimbatore.

Satpute, U. S., Patil, V. N., Katole, S. R., Men, V. D. and Thakare, A. V., 1990, Avoidable field losses due to sucking pests and bollworms in cotton. J. Appl. Zool. Res., 1 (2): $67-72$.

\section{How to cite this article:}

Vinayak Hosamani, B.M. Chittapur, Mallikarjun, A.S. Halepyati, Satyanarayana Rao, M.B. Patil, N.L. Rajesh and Venkatesh Hosamani. 2018. Effect of Site Specific Nutrient Management for Targeted Yields on Population Dynamics of Sucking Pests in Bt-Cotton (Gossypium hirsutum L.). Int.J.Curr.Microbiol.App.Sci. 7(08): 4076-4081. doi: https://doi.org/10.20546/ijcmas.2018.708.423 